\title{
Jack of all trades: thymosin $\alpha 1$ and its pleiotropy
}

\author{
Luigina Romani, ${ }^{1}$ Silvia Moretti, ${ }^{1}$ Francesca Fallarino, ${ }^{1}$ Silvia Bozza, ${ }^{1}$ Loredana Ruggeri, ${ }^{2}$ \\ Andrea Casagrande, ${ }^{1,3}$ Franco Aversa, ${ }^{2}$ Francesco Bistoni, ${ }^{1}$ Andrea Velardi, ${ }^{2}$ \\ and Enrico Garaci ${ }^{4}$
}

${ }^{1}$ Department of Experimental Medicine and Biochemical Science, University of Perugia, Perugia, Italy. ${ }^{2}$ Department of Clinical and Experimental Medicine, University of Perugia, Perugia, Italy. ${ }^{3}$ Istituto Superiore di Sanità, Roma, Italy. ${ }^{4}$ Department of Experimental Medicine and Biochemical Science, University of Tor Vergata, Rome, Italy

Address for correspondence: Luigina Romani, M.D., Ph.D., Department of Experimental Medicine and Biochemical Sciences, University of Perugia, Via del Giochetto, 06122 Perugia, Italy. Iromani@unipg.it

\begin{abstract}
Thymosin $\alpha 1$ (T $\alpha 1)$, a thymosin-related 28-mer synthetic amino-terminal acetylated peptide, has gained increasing interest in recent years, due to its pleiotropy. The peptide has been used worldwide as an adjuvant or immunotherapeutic agent to treat disparate human diseases, including viral infections, immunodeficiencies, and malignancies. The peptide can enhance T cell, dendritic cell (DC), and antibody responses, modulate cytokine and chemokine production, and block steroid-induced apoptosis of thymocytes. Its central role in modulating DC function and activating multiple signaling pathways that contribute to different functions may offer a plausible explanation for its pleiotropic action. Additionally, the ability of T $\alpha 1$ to activate the indoleamine 2,3-dioxygenase enzyme-which confers immune tolerance during transplantation and restrains the vicious circle of chronic inflammation-has been

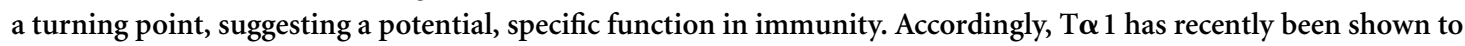
promote immune reconstitution and improve survival of recipients of HLA-matched sibling T cell-depleted stem cell transplants in a phase I/II clinical trial. Thus, T $\alpha 1$ continues to live up to its promises.
\end{abstract}

Keywords: thymosin $\alpha 1$; hematopoietic transplantation; IDO; TLRs; tolerance

\section{Introduction}

In the early 1960s, the thymus came of age with pioneering work on its importance in the development of the lymphoid system. At the same time, seminal work by Goldstein et al. led to the identification and characterization of a thymic lymphopoietic factor (thymosin $\alpha 1$ ), whose characterization was later expanded upon by Garaci's group. ${ }^{1,2}$ Pleiotropy (from the Greek $\pi \lambda \varepsilon i ́ \omega \nu$, meaning more, and $\tau \rho o \pi \eta$, meaning turn or convert) is the property of a protein to display various and sometimes opposing effects. In this regard, T $\alpha 1$, as a 28 -mer synthetic aminoterminal acetylated peptide, has been used worldwide as an adjuvant or immunotherapeutic agent to treat disparate human diseases, including viral infections, immunodeficiencies, and malignancies. $\mathrm{T} \alpha 1$ has shown an excellent safety profile and does not induce the side effects and toxicities commonly associated with immunomodulatory agents. ${ }^{3}$ Indeed, the advent of human recombinant immune modulators held promise, but the outcomes of clinical trials using biologics that target individual immune mediators have been disappointing. The complex pathophysiology of inflammatory-driven immune and autoimmune diseases, from infections to cancer, is self-amplifying and redundant at multiple levels. Thus, the intrinsic robustness of living systems against various perturbations is a key factor that prevents such compounds from being successful. This implicates that pharmacologic therapy for inflammatory diseases would only be successful if addressing these complex network systems. Any successful drug would have to be pleiotropic, and work on the many components of the inflammatory/autoimmune cascade at a time. T $\alpha 1$ exceptionally fulfills this requirement, qualifying itself as a context-dependent molecule capable of 
multitargeted interactions in physiological and nonphysiological conditions. In this review, we discuss new insights into the molecular mechanisms of T $\alpha 1$ 's pleiotropy.

\section{The multifaceted interface between T $\alpha 1$, dendritic cells, and Toll-like receptors}

The mechanism of action of T $\alpha 1$ is thought to be related to its immunomodulating activities, centered primarily on the augmentation of $\mathrm{T}$ cell function (Fig. 1). However, mechanistically, T $\alpha 1$ has shown an action beyond its effect on $\mathrm{T}$ lymphocytes to include an ability to act as an endogenous regulator of both the innate and adaptive immune systems (Fig. 2) ${ }^{4,5}$ DCs have a crucial role in determining immune outcomes by acquiring antigens, collating environmental cues, and then becoming cells that are either potent stimulators or suppressors of T cell responses. ${ }^{6}$ T $\alpha 1$ has shown the ability to modulate signals delivered through innate immune receptors on DCs, thus affecting both innate and adaptive immune responses.

One of the most basic mechanisms for activation of the DC system is through the Toll-like receptors (TLRs). TLRs belong to the type I transmembrane receptor family. Their expression is ubiquitous, from epithelial to immune cells. The TLR family mem- bers are pattern recognition receptors that collectively recognize lipid, carbohydrate, peptide, and nucleic acid structures that are broadly expressed by different groups of microorganisms. Some TLRs are expressed at the cell surface, whereas others are expressed on the membrane of endocytic vesicles or other intracellular organelles. TLR triggering induces DC maturation, which leads to the upregulation of costimulatory molecules such as CD40, CD80, and CD86, and secretion of immune modulatory cytokines and chemokines. Two major signaling pathways are generally activated in response to TLR ligands. ${ }^{7}$ One pathway involves the myeloid differentiation factor 88 (MyD88) pathway to activate nuclear factor-kappa B (NF-кB), JUN kinase (JNK), and p38, finally resulting in the production of pro-inflammatory cytokines such as tumor necrosis factor (TNF)- $\alpha$, interleukin (IL)-12, and IL-1, and induction of innate effector mechanisms. The other uses the MyD88-independent production of type I interferons via the TIR-domain-containing adapter-inducing interferon- $\beta$ (TRIF) pathway. In addition, TLRs can directly stimulate the proliferation of $\mathrm{CD}^{+}$and $\mathrm{CD} 8^{+} \mathrm{T}$ cells as well as reverse the suppressive function of $\mathrm{T}_{\text {reg }}$ cells. ${ }^{8}$

Given the ability of T $\alpha 1$ to activate TLRs, the direct responsiveness of T lymphocytes to TLR ligands

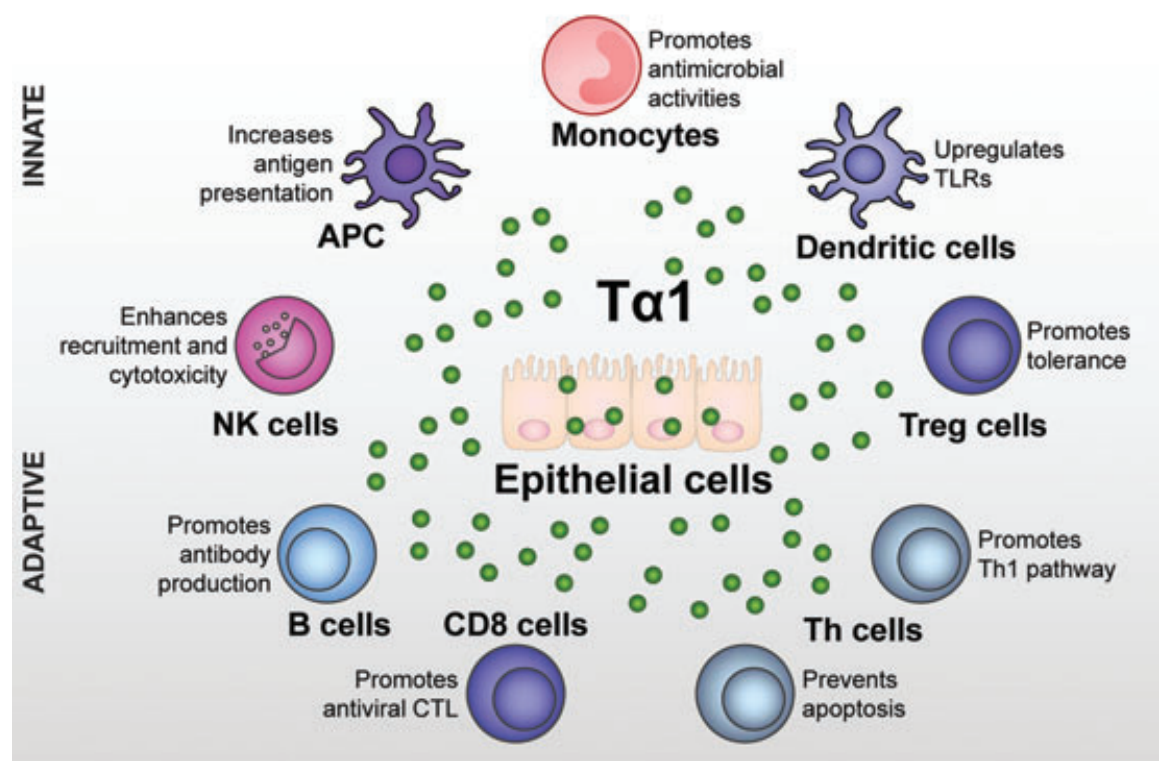

Figure 1. Pleiotropic immune activation by T $\alpha$. The drug actions of T $\alpha 1$ on cells of the innate and adaptive immune system. This is a pictorial representation of all literature-supported actions of T $\boldsymbol{\alpha} 1$ on immune cells. APC, antigen presenting cells; CTL, cytotoxic T lymphocytes; NK cells, natural killer cells; Th cells, T helper cells; $\mathrm{T}_{\text {reg }}$ cells, regulatory T cells; TLRs, Toll-like receptors. 
may offer new perspectives for the immunotherapeutic manipulation of $\mathrm{T}$ cell responses by $\mathrm{T} \alpha 1$. T $\alpha 1$ strongly upregulated the expression of TLR2 and TLR9 by murine and human DC subsets and activated NF- $\mathrm{B}$ and JNK/p38/AP-1 pathways. ${ }^{9,10}$ Induction of TLR 2 or TLR 9 expression by T $\alpha 1$ was associated with a distinct activation program in DC subsets and involved both the MyD88 and TRIF pathways. T $\alpha 1$ induced the production of IL12 p70 and IL-10 in a TLR-dependent manner. Production of IL-12p70 was reduced in TLR2 $2^{-/-}$mice, whereas IL-10 was reduced in TLR $9^{-/-}$mice. Our preliminary findings seem to suggest that the abil- ity of $\mathrm{T} \alpha 1$ to signal through disparate TLRs on DC subsets may occur through the interaction with multiple, yet distinct, TLR ligands, either endogenous or exogenous (Fig. 2). This finding not only qualifies $T \alpha 1$ as a context-oriented molecule but may also offer molecular explanations for a successful drug working on the many components of the inflammatory/immune response, that is, for successful pleiotropy.

The immunomodulatory effects of T $\alpha 1$ on DCs correlated with a therapeutic effect of the peptide in experimental fungal or viral infections. ${ }^{5}$ Administration of T $\alpha 1$ to mice with Aspergillus fumigatus

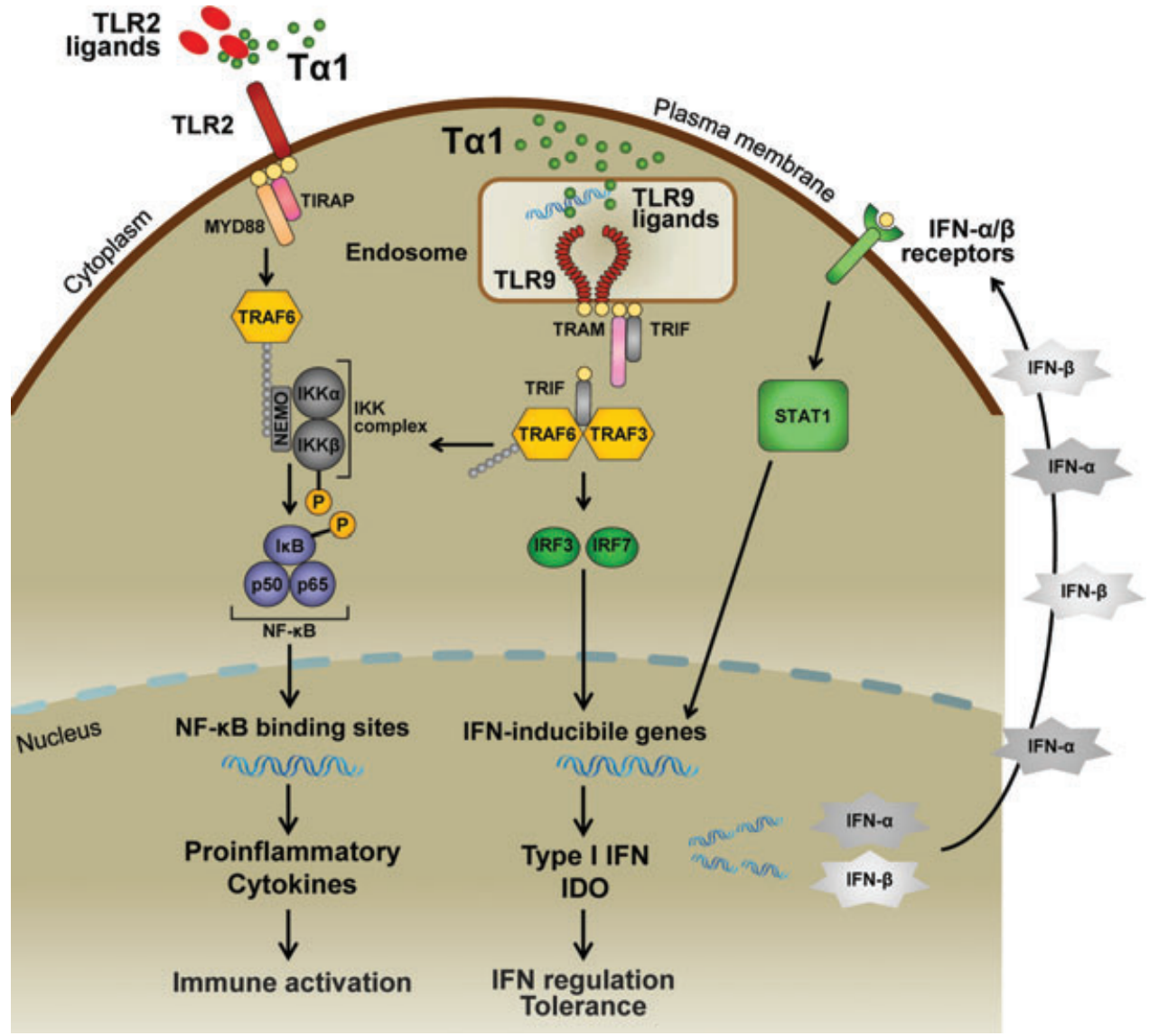

Figure 2. A view of the possible molecular mechanisms underlying T $\alpha$ 1's pleiotropy. Through heterocomplexes and collaborative interactions between endogenous and/or exogenous TLR agonists, T $\alpha 1$ may lead to the activation of different members of the NF- $\mathrm{B}$ and IRF families that plays a central role in the stress response and inflammation and transcription of interferons. Thus, the intersection between canonical and noncanonical signaling pathways may be crucial in promoting an optimally protective response balanced between inflammation and tolerance by T $\alpha$ 1. IDO, indoleamine 2,3-dioxygenase; IKK, Inhibitory kynase; IRF, Interferon regulatory factor; MyD88, myeloid differentiation factor 88; NEMO, NF- $\kappa$ B essential modulator; NF- $\kappa$, nuclear factor$\kappa B$; TRIF, TIR-domain-containing adapter-inducing interferon- $\beta$; STAT1, Signal Transducer and Activator of Transcription; TRAF, TNF receptor associated factors.; TRAM, TRIF-related adaptor molecule; TIRAP, Toll-interleukin 1 receptor domain containing adaptor protein. 
resulted in a state of full protection to the fungus as revealed by the increased survival after the infection that paralleled the reduced fungal growth and the promotion of interferon (IFN)- $\boldsymbol{\gamma}$-producing Th1 cells. ${ }^{9}$ The achievement of full protection to the fungus required the coordinated action of several TLRs on innate effector cells. Consistent with the in vitro data on TLR expression, the therapeutic efficacy of $\mathrm{T} \alpha 1$ in vivo was dependent on MyD88 signaling activated by TLR2 and partially TLR9. ${ }^{9}$ Therefore, despite a degree of redundancy in the TLR usage, the MyD88-dependent signaling pathway is essential in the antifungal activity of Tal in vitro and in vivo. In contrast, the ability of T $\alpha 1$ to modulate DC functioning through TLR9 essentially correlated with an effect on cytomegalovirus (CMV) sensing by DCs in vitro and in vivo. ${ }^{11}$ The antiviral effect of T $\alpha 1$ led to the activation of interferon regulatory factor (IRF) 7 and the promotion of the IFN- $\alpha /$ IFN- $\gamma$-dependent effector pathway in plasmacytoid DCs via the TLR9-dependent viral recognition sensing. After infection, T $\alpha 1$ decreased the viral load in mice with primary murine CMV infection and induced the expansion of cytolytic NK1. $1^{+}$cells, IFN- $\gamma$-producing $\mathrm{CD}^{+}$or $\mathrm{CD}^{+} \mathrm{T}$ cells, and the production of IL-12p70, IFN- $\alpha$, IFN- $\gamma$, and IL-10. ${ }^{11}$ Together, these data indicate that T $\alpha 1$ affects both the innate and adaptive antimicrobial immune responses in vivo.

\section{En route to tolerance: T $\alpha 1$ meets IDO and induces $T_{\text {reg }}$ cells}

The induction of tolerance is critical for the maintenance of immune homeostasis. DCs not only play a key role in the induction of immune responses, but also in the induction and maintenance of immune tolerance. ${ }^{6}$ Recent studies have demonstrated a crucial role for tryptophan catabolism and kynurenine production in the induction of peripheral tolerance. ${ }^{12,13}$ The enzyme indoleamine 2,3-dioxygenase (IDO) is an intracellular hemecontaining enzyme that catalyzes the initial and ratelimiting step in tryptophan degradation along the kynurenine pathway. IDO is widely expressed in a variety of human tissues as well as in macrophages and DCs, and is induced in inflammatory states by IFN- $\gamma$ and other proinflammatory cytokines. Work has demonstrated a complex and crucial role for IDO in immunoregulation during infection, pregnancy, autoimmunity, transplantation, and neopla- sia. ${ }^{13}$ TLR9 stimulation may lead to the activation of the tryptophan catabolism pathway in DCs, ${ }^{14}$ and IDO is a molecular signature of tolerogenic DCs. ${ }^{15}$ Through localized tryptophan deficiency combined with the release of proapoptotic metabolites, DCs can thus exert an IDO-dependent homeostatic control over the proliferation and survival of peripheral T cells, and can promote antigen-specific tolerance.

T $\alpha 1$ was found to induce IDO activity in DCs, and this affected Th priming and tolerization. IDO induction required TLR9 and type I IFN receptor signaling and resulted in IL-10 production. ${ }^{16}$ The induction of IDO by T $\alpha 1$ affected the relative ability of DCs to balance antigen-specific $\mathrm{Th} / \mathrm{T}_{\text {reg }}$ cell priming in vitro and in vivo. T $\alpha 1$ increased priming for IFN- $\gamma-$ and IL-10-producing $\mathrm{CD} 4^{+}$ $\mathrm{T}$ cells, that is, it induced $\mathrm{Th} 1 / \mathrm{T}_{\text {reg }}$ cell activation. IDO blockade prevented the activation of IL-10 producing $\mathrm{CD}^{+}$cells, while sparing that of IFN$\gamma$-producing cells, a finding confirming the causal link of IDO with priming for IL-10-producing T cells. Consistent with the finding that TLR9 stimulation can promote DC-mediated generation of $\mathrm{CD} 4{ }^{+} \mathrm{CD} 25^{+} \mathrm{T}_{\text {reg }}$ cells, ${ }^{17} \mathrm{CD} 4{ }^{+} \mathrm{CD} 25^{+} \mathrm{T}$ cells induced by T $\alpha 1$-treated DCs expressed FoxP $3^{9,16}$ that is crucially involved in the suppressive phenotype of $\mathrm{T}_{\text {reg }}$ cells. Therefore, $\mathrm{T} \alpha 1$ induced $\mathrm{CD} 4^{+} \mathrm{CD} 25^{+} \mathrm{T}_{\text {reg }}$ cells through the activation of a TLR9-dependent pathway.

\section{Boosting tolerance by $\mathrm{T} \alpha 1$}

Within the new paradigm of resistance and tolerance mechanisms as two alternative but complementary host defense strategies, ${ }^{18}$ boosting tissue tolerance is likely to be a useful strategy in infectious and noninfectious diseases. The mammalian intestinal and respiratory mucosal surfaces are continuously exposed to a complex and dynamic community of microorganisms. These microbes establish symbiotic relationships with their hosts, making important contributions to immune fitness and metabolism efficiency. ${ }^{19,20}$ The immune system is pivotal in mediating the interactions between host and microbiota that shape the mucosal environment. At the mucosal surfaces, a system of tolerance and controlled inflammation to limit the response to dietary or pathogen-derived antigens is present. This regulation is achieved by a number of cell populations acting through a set of shared regulatory 
pathways. ${ }^{21}$ Current knowledge holds that populations of suppressor $\mathrm{T}_{\text {reg }}$ cells constitute a pivotal mechanism of immunological tolerance. The potential role of malfunctioning $\mathrm{T}_{\text {reg }}$ cells in chronic inflammatory immune and autoimmune diseases is well documented. Learning how to successfully manipulate $\mathrm{T}_{\text {reg }}$ cell responses could result in more effective vaccines and immunomodulators. Indeed, dysfunction of the innate and adaptive immune systems associated with mucosa causes impairment of mucosal barrier function and development of localized or systemic inflammatory and autoimmune processes. When this complex system breaks down in a genetically predisposed individual, the resulting immune response my lead to localized and systemic inflammatory diseases. ${ }^{22,23}$ By inducing IDO and promoting $\mathrm{T}_{\text {reg }}$ cells, $\mathrm{T} \alpha 1$ successfully ameliorated respiratory allergy and intestinal inflammation in different experimental models. ${ }^{4,5}$ Thus, maintaining diplomatic relations between mammals and beneficial microbial communities could be added to the therapeutic and immunomodulatory properties of $\mathrm{T} \alpha 1$.

\section{T $\alpha 1$ promotes immunity and tolerance in hematopoietic transplantation}

The reconstitution of immunity in the recipients remains the most challenging aspect of hematopoietic stem cell transplantation (HSCT). Successful T cell reconstitution needs to accommodate the induction of protective immunity to pathogens and tumors within the context of transplantation tolerance. This implicates the requirement of DCs capable of generating some form of dominant regulation that ultimately controls inflammation, pathogen immunity and tolerance. T $\alpha 1$-primed DCs fulfill these multiple requirements, which include the induction of priming to pathogens and tolerance to alloantigens in experimental and human HSCT. In experimental HSCT, the infusion of T $\alpha 1$-treated DCs resulted in pathogen clearance and prevention of graft-versus host disease (GVHD). ${ }^{16}$ Thus, T $\alpha 1$ acted as the ideal immunomodulator capable of antimicrobial priming and concomitant tolerance in HSCT. Accordingly, T $\alpha 1$ has recently been shown to promote immune recipients of HLA-matched sibling $\mathrm{T}$ cell-depleted stem cell transplants in a phase I/II clinical trial presented by Ruggeri et al. ${ }^{24} \mathrm{Re}-$ markably, T $\alpha 1$ also improved survival, as the cumulative incidence of nonrelapse mortality (NRM, mainly infection-related) was $32 \%$ in controls versus $7 \%$ in T $\alpha 1$-treated patients. Multivariable analyses that included diagnoses, disease status at transplant, conditioning regimen and donor lymphocyte infusions showed that $\mathrm{T} \alpha 1$ treatment was a significant independent factor predicting a lower incidence of NRM, which tended to provide better survival. Thus, T $\alpha 1$ continues to live up to its promises.

\section{Balancing immunity and tolerance: learning from $T \alpha 1$}

Through a multifaceted, pleiotropic immune activation, T $\alpha 1$ appears to be a promising adjuvant candidate and is suitable for combination therapies aimed at the control of inflammation, immunity, and tolerance in a variety of clinical settings. In addition to living up to its promises, T $\alpha 1$ continues to amaze us with its pleiotropy, multitargeting activity, and ability to utilize different molecular mechanisms depending on contexts. Heterocomplexes and collaborative interactions between endogenous and/or exogenous TLR agonists and T $\alpha 1$ appears to lead to novel reciprocal trends of immune surveillance and effector mechanisms aiding safe disposal of pathogens and/or pathogenic insults whereby excessive inflammatory responses and tissue injury may effectively be prevented (Fig. 2). NF- $\mathrm{BB}$, a family of seven structurally related transcription factors that play a central role in the stress response and inflammation $^{25,26}$ are likely exploited by $\mathrm{T} \alpha 1$ for its pleiotropic activity. Although the NF- $\kappa \mathrm{B}$ subunits are ubiquitously expressed, their actions are regulated in a cell type- and stimulus-specific manner, allowing for a diverse spectrum of effects. Molecular dissection of its mechanisms for activation has shown that NF- $\mathrm{B}$ can be induced by the "canonical" and "noncanonical" pathways, leading to distinct patterns in the individual subunits activated and downstream genetic responses produced. Much attention has been focused on the proinflammatory signaling of canonical NF- $\kappa \mathrm{B}$, but data also indicate that noncanonical NF- $\kappa \mathrm{B}$ could have opposing roles, limiting canonical NF- $\kappa \mathrm{B}$ activity, inducing IDO, and controlling inflammation (Fig. 2). ${ }^{27}$ Thus, the intersection between canonical and noncanonical NF- $\kappa \mathrm{B}$ signaling pathways and IRFs may be crucial in promoting an optimally protective response balanced between inflammation and tolerance by $\mathrm{T} \alpha 1$. 


\section{Acknowledgments}

We thank Dr. Cristina Massi Benedetti for digital art and editing. The original studies conducted in the author's laboratory were supported by the Italian Projects AIDS 2010 by the Istituto Superiore di Sanità (contract number $40 \mathrm{H} 40$ to L.R.) and by the Specific Targeted Research Project ALLFUN (FP7HEALTH-2009 contract number 260338 to L.R.).

\section{Conflicts of interest}

The authors declare no conflicts of interest.

\section{References}

1. Goldstein, A.L. 2009. From lab to bedside: emerging clinical applications of thymosin alpha 1. Expert. Opin. Biol. Ther. 9: 593-608.

2. Garaci, E. et al. 2000. Thymosin alpha 1 in the treatment of cancer: from basic research to clinical application. Int. J. Immunopharmacol. 22: 1067-1076.

3. Billich, A. 2002. Thymosin alpha1. SciClone Pharmaceuticals. Curr. Opin. Investig. Drugs 3: 698-707.

4. Pierluigi, B. et al. 2010. Thymosin alphal: the regulator of regulators? Ann. N.Y. Acad. Sci. 1194: 1-5.

5. Romani, L. et al. 2007. Thymosin alpha1: an endogenous regulator of inflammation, immunity, and tolerance. Ann. N.Y. Acad. Sci. 1112: 326-338.

6. Steinman, R.M., D. Hawiger \& M.C. Nussenzweig. 2003. Tolerogenic dendritic cells. Annu. Rev. Immunol. 21: 685711.

7. Kawai, T. \& Akira, S. 2011. Toll-like receptors and their crosstalk with other innate receptors in infection and immunity. Immunity 34: 637-650.

8. Kabelitz, D. 2007. Expression and function of Toll-like receptors in T lymphocytes. Curr. Opin. Immunol. 19: 39-45.

9. Romani, L. et al. 2004. Thymosin alpha 1 activates dendritic cells for antifungal Th1 resistance through toll-like receptor signaling. Blood 103: 4232-4239.

10. Zhang, P. et al. 2005. Activation of IKK by thymosin alpha1 requires the TRAF6 signalling pathway. EMBO Rep. 6: 531537.

11. Bozza, S. et al. 2007. Thymosin alphal activates the TLR9/MyD88/IRF7-dependent murine cytomegalovirus sensing for induction of anti-viral responses in vivo. Int. Immunol. 19: 1261-1270.
12. Grohmann, U., F. Fallarino \& P. Puccetti. 2003. Tolerance, DCs and tryptophan: much ado about IDO. Trends Immunol. 24: 242-248.

13. Mellor, A.L. \& D.H. Munn. 2004. IDO expression by dendritic cells: tolerance and tryptophan catabolism. Nat. Rev. Immunol. 4: 762-774.

14. Fallarino, F. \& P. Puccetti. 2005. Toll-like receptor 9-mediated induction of the immunosuppressive pathway of tryptophan catabolism. Eur. J. Immunol. 36: 8-11.

15. Orabona, C. et al. 2006. Toward the identification of a tolerogenic signature in IDO-competent dendritic cells. Blood 107: 2846-2854.

16. Romani, L. et al. 2006. Thymosin alphal activates dendritic cell tryptophan catabolism and establishes a regulatory environment for balance of inflammation and tolerance. Blood 108: 2265-2274.

17. Moseman, E.A. et al. 2004. Human plasmacytoid dendritic cells activated by $\mathrm{CpG}$ oligodeoxynucleotides induce the generation of CD4+CD25+ regulatory T cells. J. Immunol. 173: 4433-4442.

18. Medzhitov, R., D.S. Schneider \& M.P. Soares. 2012. Disease tolerance as a defense strategy. Science 335: 936-941.

19. Hooper, L.V. \& A.J. Macpherson. 2010. Immune adaptations that maintain homeostasis with the intestinal microbiota. Nat. Rev. Immunol. 10: 159-169.

20. Huang, Y.J. \& S.V. Lynch. 2011. The emerging relationship between the airway microbiota and chronic respiratory disease: clinical implications. Expert. Rev. Respir. Med. 5: 809821.

21. Saraiva, M. \& A. O'Garra. 2010. The regulation of IL-10 production by immune cells. Nat. Rev. Immunol. 10: 170181.

22. Clemente, J.C. et al. 2012. The impact of the gut microbiota on human health: an integrative view. Cell 148: 1258-1270.

23. Cho, I. \& M.J. Blaser. 2012. The human microbiome: at the interface of health and disease. Nat. Rev. Genet. 13: 260-270.

24. Perruccio, K. et al. 2010. Thymosin alpha1 to harness immunity to pathogens after haploidentical hematopoietic transplantation. Ann. N.Y. Acad. Sci. 1194: 153-161.

25. Hayden, M.S. \& S. Ghosh. 2004. Signaling to NF-kappaB. Genes Dev. 18: 2195-2224.

26. Bonizzi, G. \& M. Karin. 2004. The two NF-kappaB activation pathways and their role in innate and adaptive immunity. Trends Immunol. 25: 280-288.

27. Grohmann, U. et al. 2007. Reverse signaling through GITR ligand enables dexamethasone to activate IDO in allergy. Nat. Med. 13: 579-586. 